\title{
A Color-Texture Based Segmentation Method To Extract Object From Background
}

\author{
Saka Kezia \\ Assoc. Professor, Dept. of ECE, CIET Rajahmundry, JNTUK, A.P, INDIA \\ E-mail: sakakezia1981@gmail.com \\ Dr. I. Santi Prabha \\ Professor, Dept. of ECE, JNTUCE, JNTUK, Kakinada, A.P, INDIA \\ E-mail: santiprabha@yahoo.com \\ Dr. V. Vijaya Kumar \\ Dean Dept. of CSE \& IT, MCA, GIET, Rajahmundry, JNTUK, A.P, INDIA \\ E-mail: vijayvakula@yahoo.com
}

\begin{abstract}
Extraction of flower regions from complex background is a difficult task, it is an important part of flower image retrieval, and recognition .Image segmentation denotes a process of partitioning an image into distinct regions. A large variety of different segmentation approaches for images have been developed. Image segmentation plays an important role in image analysis. According to several authors, segmentation terminates when the observer's goal is satisfied. For this reason, a unique method that can be applied to all possible cases does not yet exist. This paper studies the flower image segmentation in complex background. Based on the visual characteristics differences of the flower and the surrounding objects, the flower from different backgrounds are separated into a single set of flower image pixels. The segmentation methodology on flower images consists of five steps. Firstly, the original image of RGB space is transformed into Lab color space. In the second step ' $a$ ' component of Lab color space is extracted. Then segmentation by two-dimension OTSU of automatic threshold in 'achannel' is performed. Based on the color segmentation result, and the texture differences between the background image and the required object, we extract the object by the gray level co-occurrence matrix for texture segmentation. The GLCMs essentially represent the joint probability of occurrence of grey-levels for pixels with a given spatial relationship in a defined region. Finally, the segmentation result is corrected by mathematical morphology methods. The algorithm was tested on plague image database and the results prove to be satisfactory. The algorithm was also tested on medical images for nucleus segmentation.
\end{abstract}

Index Terms - Color image segmentation, Morphology, OTSU Thresholding, GLCM

\section{INTRODUCTION}

Color image segmentation is useful in many applications. From the segmentation results, it is possible to identify regions of interest and objects in the scene, which is very beneficial to the subsequent image analysis or annotation. Recent work includes a variety of techniques: for example, stochastic model based approaches [1], [2], [3], [4], [5], morphological watershed based region growing [6], energy diffusion [7], and graph partitioning [8]. Quantitative evaluation methods have also been suggested [9]. However, due to the difficult nature of the problem, there are few automatic algorithms that can work well on a large variety of data. The problem of segmentation is difficult because of image texture. If an image contains only homogeneous color regions, clustering methods in color space such as [10] are sufficient to handle the problem. In reality, natural scenes are rich in color and texture. It is difficult to identify image regions containing colortexture patterns.

Flowers in images are surrounded by a green or brown background and need to be segmented from the background. This paper presents new color and texture based method for segmenting flowers. The colored flowers are segmented from the background. Developing a system for segmentation of flowers is a difficult task. In real environment, images of flowers are often taken in natural outdoor scenes where the lighting condition varies with the weather and time. In addition, flowers are often more or less transparent and specula highlights can make the flower appear light or even white causing the illumination problem. In addition, there is lot more variation in viewpoint, occlusions, scale of flower images. All these problems lead to a confusion and make the task of flower segmentation more challenging. In addition, the background also makes the problem difficult, as a flower has to be segmented automatically.

Applications of segmentation of flowers can be found useful in floriculture, flower searching for patent analysis etc. The floriculture has become one of the important commercial trades in agriculture owing to steady increase in demand of flowers. Floriculture industry comprises of flower trade, nursery and potted plants, seed and bulb production, micro propagation and extraction of essential oil from flowers. In such cases, 
automation of flower segmentation is very essential. Flower segmentation can be used in flower recognition. Further, flower recognition is used for searching patent flower images to know whether the flower image applied for patent is already present in the patent image database or not [11]. Since these activities are being done manually and it is mainly labor dependent and hence automation is necessary.

Nilsback and Zisserman [12] proposed a two-step model to segment the flowers in color images, one to separate foreground and background and another model to extract the petal structure of the flower. This segmentation algorithm is tolerant to changes in viewpoint and petal deformation, and the method is applicable in general for any flower class. Das et al., [13] proposed an indexing method to index the patent images using the domain knowledge. The flower was segmented using iterative segmentation algorithm with the domain knowledge driven feedback. A threshold-based segmentation was proposed in [14].The paper in [15] presented a segmentation technique based on anisotropic diffusion, which is less complex. The colored flowers are segmented from the background. In this diffusion technique, the edge stopping function is computed as inversely proportional to the weighted sum of gradients of color components.

The present paper is organized as follows: section II deals with the basic concepts of mathematical morphology, section III introduces GLCM, section IV deals with OTSU thresholding, section V describes the proposed methodology, in section VI the experimental results are discussed and finally section VII deals with conclusions.

\section{MATHEMATICAL MORPHOLOGY}

Mathematical morphology is a set theory approach, developed by J.Serra and G. Matheron. It provides an approach to processing of digital images that is based on geometrical shape. Two fundamental morphological operations - erosion and dilation are based on Minkowski operations. There are two different types of notations for these operations: Serra/Matheron notation and Haralick/Sternberg notation. In this paper Haralick/Sternberg notation, which is probably more often used in practical applications, is used. In this notation, erosion is defined as follows (Eq. 1) (Serra, 1982):

$$
X \Theta B=\bigcap_{y \in B} X_{y}
$$

and dilation (Eq. 2) as:

$$
\begin{aligned}
& X \oplus B=\bigcup_{y \in B} X y \\
& X y=\{x+y: x \in X\}
\end{aligned}
$$

$$
\hat{B}=\{b:-b \in B\}
$$

Another important pair of morphological operations are opening and closing. They are defined in terms of dilation and erosion, by equations (5) and (6) respectively.

$$
\begin{aligned}
& X \circ B=(X \Theta B) \oplus B \\
& X \bullet B=(X \oplus B) \Theta B
\end{aligned}
$$

Morphological filling is another important operation used to fill holes in an image. Let ' $A$ ' denote a set whose elements are 8- connected boundaries, each boundary enclosing a background region i.e., a hole. Given a point in each hole, the objective is to fill all the holes with $1 \mathrm{~s}$. The initial array $\mathrm{X}_{0}$ is formed with $0 \mathrm{~s}$ and $1 \mathrm{~s}$ and is of same size as of ' $A$ '. The value ' 1 ' in $X_{0}$ corresponds to the given point in each hole. Filling operation is defined by equation (7) as

$X_{k}=\left(X_{k-1} \oplus B\right) \cap A^{c}$

where $\mathrm{B}$ is the structuring element. The algorithm terminates at iteration step $k$ if $X_{k}=X_{k-1}$. The set $X_{k}$ contains all the filled holes. The set union of $X_{k}$ and $A$ contains all the filled holes and their boundaries.

\section{GLCM}

Gray level co-occurrence matrix (GLCM) has been proven to be a very powerful tool for texture image segmentation [16, 17]. The only shortcoming of the GLCM is its computational cost. Such restriction causes impractical implementation for pixel-by-pixel image processing. In the previous works, GLCM computational burden was reduced by two methods, at the computation architecture level and hardware level. D. A. Clausi et. al. restructures the GLCM by introducing a GLCLL (gray level co-occurrence linked list), which discard the zero value in the GLCM [18].This technique gives a good improvement because mostly GLCM is a sparse matrix where most of its values are equal to zeroes. Thus, the size of GLCLL is significantly smaller than GLCM. Then the structure of the GLCLL was improved in [19, 20]. Another work is presented in [21] where fast calculation of GLCM texture features relative to a window spanning an image in a raster manner was introduced. This technique was based on the fact that windows relative to adjacent pixels are mostly overlapping, thus the features related to the pixels inside the overlapping windows can be obtained by updating the early calculated values. In January 2007, S. Kiranyaz and M. Gabbouj proposed a novel indexing technique called Hierarchical Cellular Tree (HCT) to handle large data [22]. In his work, it was proved that the proposed technique is able to reduce the GLCM texture features computation burden.

where:

B and $\hat{B}$ are structuring elements 


$$
\begin{aligned}
C_{m, n, \phi}=\sum_{x} \sum_{y} P\{I(x, y)=m \\
\left.\quad \& I\left(x \pm d \phi_{0}, y \mp d \phi_{1}\right)=n\right\}
\end{aligned}
$$

GLCM is a matrix that describes the frequency of one gray level appearing in a specified spatial linear relationship with another gray level within the area of investigation [23]. Here, the co-occurrence matrix is computed based on two parameters, which are the relative distance between the pixel pair $d$ measured in pixel number and their relative orientation $\varphi$. Normally, $\varphi$ is quantized in four directions $\left(0^{0}, 45^{\circ}, 90^{\circ}\right.$ and $\left.135^{\circ}\right)$ [23]. In practice, for each $d$, the resulting values for the four directions are averaged out. To show how the computation is done, for image $\mathrm{I}$, let $\mathrm{m}$ represent the gray level of pixels $(\mathrm{x}, \mathrm{y})$ and $\mathrm{n}$ represent the gray level of pixels $\left(\mathrm{x} \pm \mathrm{d} \phi_{0}, \mathrm{y} \pm \mathrm{d} \phi_{1}\right)$ with $\mathrm{L}$ level of gray tones where $0 \leq \mathrm{x} \leq \mathrm{M}-1,0 \leq \mathrm{y} \leq \mathrm{N}-1$ and $0 \leq \mathrm{m}, \mathrm{n} \leq \mathrm{L}-1$. From these representations, the gray level co-occurrence matrix $\mathrm{Cm}$, $\mathrm{n}$ for distance $\mathrm{d}$ and direction $\phi$ can be defined as (8)

Where $\mathrm{P}\{\}=$.1 if the argument is true and otherwise, $\mathrm{P}\{\}=$.0 . For each $\phi$ value, its $\phi_{0}$ and $\phi_{1}$ values are referred as in the Table 1.

Table 1: Orientation constant

\begin{tabular}{|l|l|l|}
\hline$\phi$ & $\phi_{0}$ & $\phi_{1}$ \\
\hline $0^{0}$ & 0 & 1 \\
\hline $45^{0}$ & -1 & -1 \\
\hline $90^{0}$ & 1 & 0 \\
\hline $135^{0}$ & 1 & -1 \\
\hline
\end{tabular}

In the classical paper [24], Haralick et. al introduced fourteen textural features from the GLCM and then in [25] stated that only six of the textural features are considered to be the most relevant. Those textural features are Energy, Entropy, Contrast, Variance, Correlation and Inverse Difference Moment. Energy is also called Angular Second Moment (ASM) where it measures textural uniformity [23]. If an image is completely homogeneous, its energy will be maximum. Entropy is a measure, which is inversely correlated to energy. It measures the disorder or randomness of an image [23]. Next, contrast is a measure of local gray level variation of an image. This parameter takes low value for a smooth image and high value for a coarse image. On the other hand, inverse difference moment is a measure that takes a high value for a low contrast image. Thus, the parameter is more sensitive to the presence of the GLCM elements, which are nearer to the symmetry line $\mathrm{C}(\mathrm{m}, \mathrm{m})$ [23]. Variance as the fifth parameter is a measure that is similar to the first order statistical variables called standard deviation [26]. The last parameter, correlation, measures the linear dependency among neighboring pixels. It gives a measure of abrupt pixel transitions in the image [27].

\section{IV.OTSU THRESHOLDING}

This method, as proposed by [25] is based on discriminate analysis. The threshold operation is regarded as the partitioning of the pixels of an image into two classes $\mathrm{C} 0$ and $\mathrm{C} 1$ (e.g., objects and background) at grey-level t, i.e., $\mathrm{C} 0=\{0,1,2, \mathrm{t}\}$ and $\mathrm{C} 1$ $=\{\mathrm{t}+1, \mathrm{t}+2, \ldots \mathrm{L}-1\}$. Let $\sigma 2 \mathrm{w}, \sigma 2 \mathrm{~B}$ and $\sigma 2 \mathrm{~T}$ be the within-class variance, between-class variance, and the total variance, respectively. An optimal threshold can be determined by minimizing one of the following (equivalent) criterion functions with respect to

$$
\lambda=\frac{\sigma_{B}^{2}}{\sigma_{W}^{2}}, \eta=\frac{\sigma_{B}^{2}}{\sigma_{T}^{2}}, \quad \kappa=\frac{\sigma_{T}^{2}}{\sigma_{W}^{2}}
$$

The optimal threshold $\mathrm{t}$ is defined as

$\mathrm{t}=\operatorname{ArgMin} \eta$

$\sigma_{T}^{2}=\sum_{i=0}^{L-1}\left[1-\mu_{T}\right]^{2} P_{i}, \mu_{T}=\sum_{i=0}^{L-1}\left[i P_{i}\right]$

$\sigma_{B}^{2}=W_{0} W_{1}\left(\mu_{0} \mu_{1}\right)^{2}$

$$
\begin{aligned}
& W_{0}=\sum_{i=0}^{t} P_{i}, \quad W_{1}=1-W_{0} \\
& \mu_{1}=\frac{\mu_{T}-\mu_{t}}{1-\mu_{0}}, \mu_{0}=\frac{\mu_{t}}{W_{0}}, \mu_{t}=\sum_{i=0}^{t}\left(i P_{i}\right) \\
& P_{i}=\frac{n_{i}}{n} \\
& n=\sum_{i=0}^{L-1} n_{i}
\end{aligned}
$$

Moreover, $\mathrm{P}_{\mathrm{i}}$ is the probability of occurrence of greylevel i. For a selected threshold ' $t$ ' of a given image, the class probabilities $\mathrm{w}_{0}$ and $\mathrm{w}_{1}$ indicate the portions of the areas occupied by the classes $\mathrm{C}_{0}$ and $\mathrm{C}_{1}$. The class means $\mu_{0}$ and $\mu_{1}$ serve as estimates of the mean levels of the classes in the original grey-level image. Moreover, the maximum value of $\eta$, denoted by $\eta^{*}$, can be used as a measure to evaluate the separability of classes $\mathrm{C}_{0}$ and $\mathrm{C}_{1}$ in the original image or the bimodality of the histogram. This is a very significant measure because it is invariant under affine transformations of the greylevel scale. It is uniquely determined within the range $0 \leq \eta \leq 1$. The lower bound (zero) is obtained when and only when a given image has a single constant grey level, and the upper bound (unity) is obtained when and only when two-valued images are given.

\section{V.METHODOLOGY}

In the first step RGB image is converted to Lab color space. Lab color space is a three-dimensional space map based on the composition of chroma and luminance. In this model $\mathrm{L}$ denotes the luminance, $\mathrm{a}$ and $\mathrm{b}$ components 
represents the chroma value. The solution to convert digital images from the RGB space to the $\mathrm{L}^{*} \mathrm{a} * \mathrm{~b} *$ color space which had been mentioned below in the following formula

$$
\left.\begin{array}{l}
\mathrm{L}^{*}=116 \mathrm{f}(\mathrm{Y} / \mathrm{Yn})-16 \\
\mathrm{a}^{*}=500[\mathrm{f}(\mathrm{X} / \mathrm{Xn})-\mathrm{f}(\mathrm{Y} / \mathrm{Yn})] \\
\mathrm{b}^{*}=200[\mathrm{f}(\mathrm{Y} / \mathrm{Yn})-\mathrm{f}(\mathrm{Z} / \mathrm{Zn})]
\end{array}\right\}
$$

$\mathrm{X}, \mathrm{Y}, \mathrm{Z}, \mathrm{Xn}, \mathrm{Yn}$, and $\mathrm{Zn}$ are the coordinates of CIEXYZ color space. The solution to convert digital images from the RGB space to the CIEXYZ color space is as the following formula

$$
\left[\begin{array}{c}
\mathrm{X} \\
\mathrm{Y} \\
\mathrm{Z}
\end{array}\right]=\left[\begin{array}{ccc}
0.608 & 0.174 & 0.201 \\
0.299 & 0.587 & 0.114 \\
& & \\
0.000 & 0.068 & 1.117
\end{array}\right]\left[\begin{array}{l}
\mathrm{R} \\
\mathrm{G} \\
\mathrm{B}
\end{array}\right]
$$

$\mathrm{Xn}, \mathrm{Yn}$, and $\mathrm{Zn}$ are respectively corresponding to the white value of the parameter.

$f(x)= \begin{cases}x^{1 / 3} & x>0.008856 \\ 7.787 x+16 / 116 & x<=0.008856\end{cases}$

Each color space has its own appear background and application region. When segmenting a color image, the selection of color space plays a decisive role on the segmentation results. The common color spaces used in color image processing include RGB color space, HSI color space, Lab color space, and so on. At present, the general color digital images are RGB format. RGB color space is based on the theory of three-basic color to build. RGB format is the most basic color space. Other color space models can be obtained through the RGB format conversion. But the RGB color space is not a homogeneous visual perception space, it is not conducive to image segmentation based on color feature. HSI color space uses color characteristics of a direct sense of the three quantities: the brightness or lightness $(\mathrm{I})$, hue $(\mathrm{H})$, saturation $(\mathrm{S})$ to describe the color. This method is more in line with the human eye habits to the description of the color, but the expressed colors are incomplete visual perceived color. The Lab color space is a homogeneous space for visual perception, the difference between two points in the Lab color space is same with the human visual system. It applies to represent and calculate of all light color or object color, so the Lab color space is very effective in color image analysis.

The ' $a$ ' component is extracted from the transformed $\mathrm{Lab}$ color space in the second step. The positive direction of a-axis represents the red color's changes and the negative direction of a-axis represents the green color's changes. The 'a' channel is selected in order to eliminate the green leaves and to extract the flowers. In the third step the extracted a- component is subjected to OTSU thresholding. The two-dimensional OTSU algorithm automatically selects the optimal threshold for segmentation. Because two-dimensional OTSU algorithm not only takes into account the grayscale information of pixels, but also considers the spacerelated information of pixels and their neighborhoods. GLCM is obtained for the otsu thresholded image. For each image and with distance set to one, four GLCMs having directions $0^{\circ}, 45^{\circ}, 90^{\circ}$ and $135^{\circ}$ are generated. The co-occurrence matrix is often correlated with the directions. The directions of flowers are random. Therefore, it is difficult to describe precisely the texture feature for a flower image simply with one-direction of co-occurrence matrix. It is necessary to select more than one direction of gray level co-occurrence matrix for a comprehensive statistical processing. The synthetic gray level co-occurrence matrix of an image can be got by averaging the values of energy matrices in 0 degree, 45 degree, 90 degree and 135 degree. In the fifth step morphological filling operation is performed in order to fill small holes. A hole is defined as an area of dark pixels surrounded by lighter pixels or may be defined as a background region surrounded by a connected border of foreground pixels. This process can be used to make objects in an image seem to disappear as they are replaced with values that blend in with the background area. This function is useful for image editing, including removal of extraneous details or artifacts. As last step morphological opening operation is performed to remove small objects.

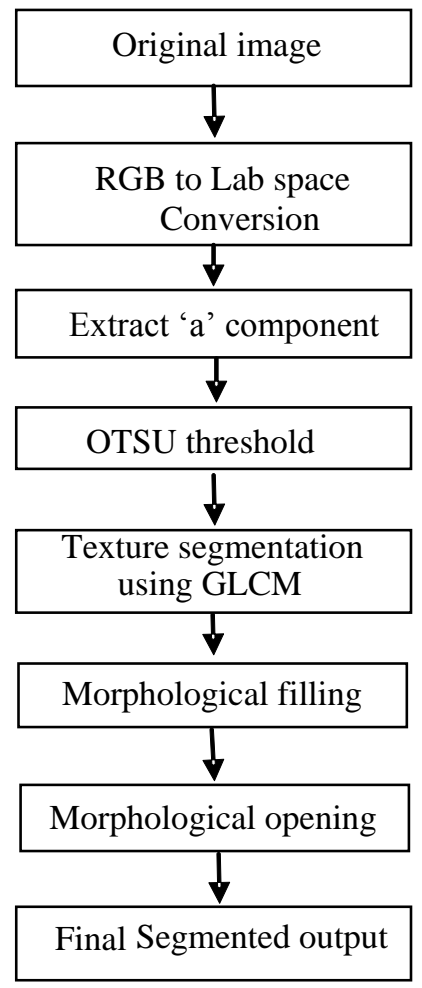

Figure 1. Flowchart of Proposed algorithm

\section{VI.RESULTS}

The proposed algorithm is tested on flower texture images of plague database. In this paper step wise results of flower images and medical images are shown in Figs.2-5. The results in Figs.2-3 clearly show the 
extraction of flower from the background. The algorithm is also tested on medical images for the extraction of nucleus in actin images and the results are shown in Figs.4-5. Actin images are collected from [28]. The results clearly indicate that the green and yellow backgrounds in the images are converted to black in the ' $a$ ' component of the image. Otsu threshold is applied for enhancing the 'a' component output. The Figs.2-5 (d) shows that the object is smoothened after texture segmentation. The Figs.2-5 (e) clearly shows that morphological filling step is applied to fill small gaps in the object. To remove small objects morphological opening step is applied as shown in Figs .2-5 (f). The results of the above algorithm applied on the medical images show that the algorithm can be used for nucleus extraction as shown in Figs 4-5.

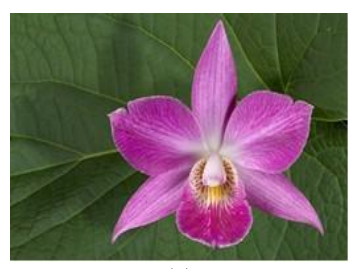

(a)

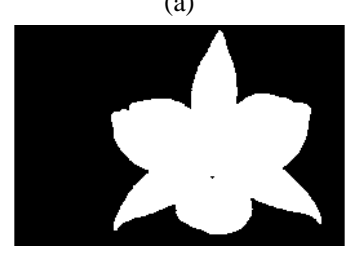

(c)

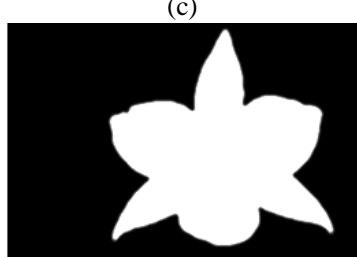

(e)

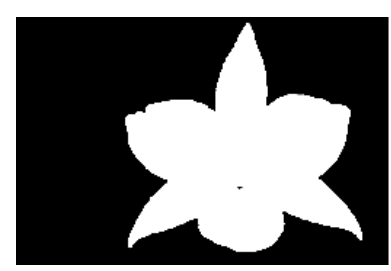

(b)

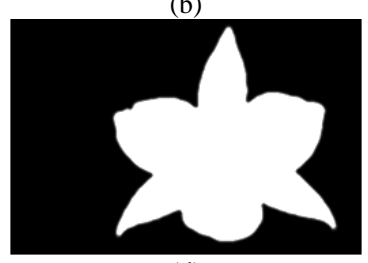

(d)

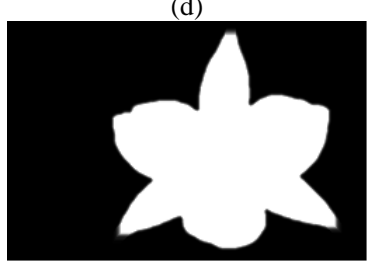

(f)

Figure 2. flower image segmentation stepwise results of proposed algorithm. (a) Original image (b) 'a' component (c) OTSU thresholded (d) GLCM output (e) morphological filling (f) opening

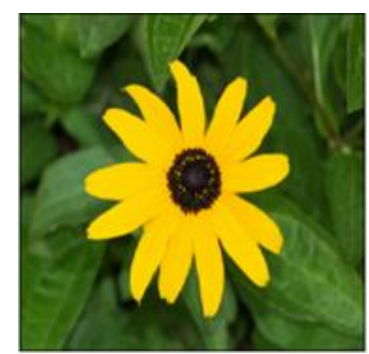

(a)

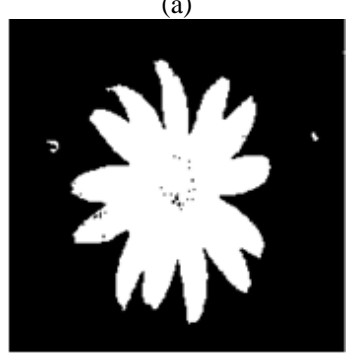

(c)

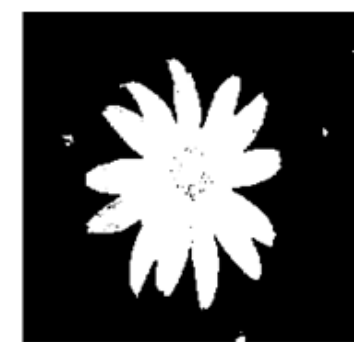

(b)

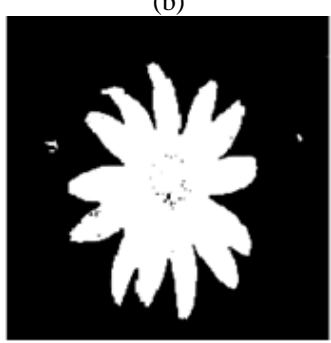

(d)

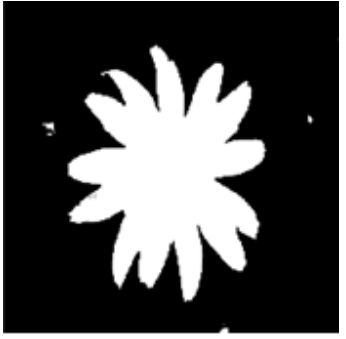

(e)

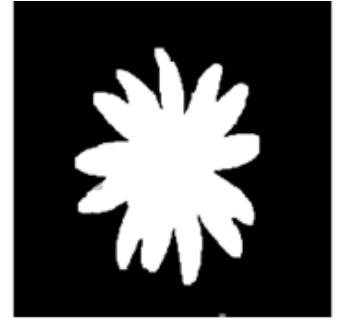

(f)

Figure 3. flower image segmentation stepwise results of proposed algorithm. (a) Original image (b) 'a' component (c) OTSU thresholded (d) GLCM output (e) morphological filling (f) opening

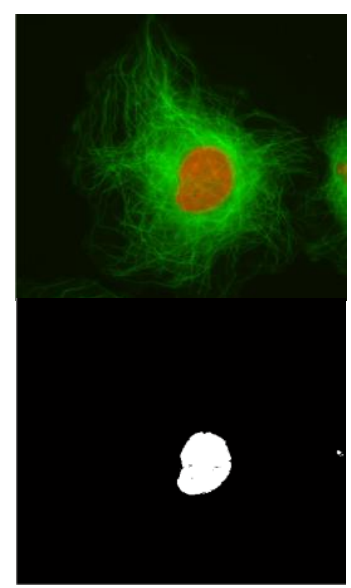

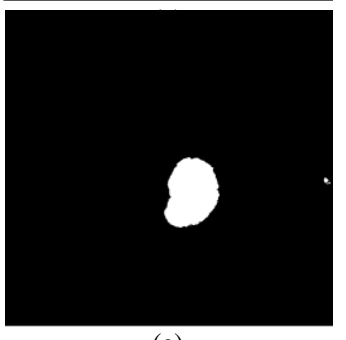

(e)

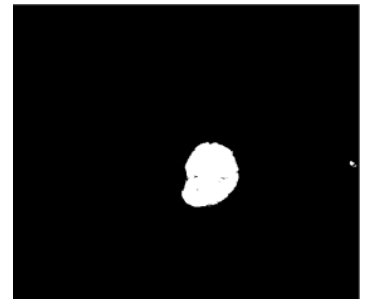

(b)

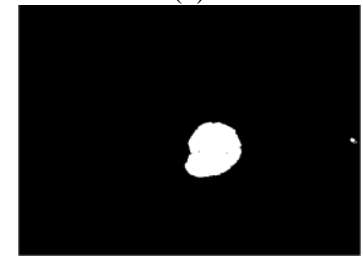

(d)

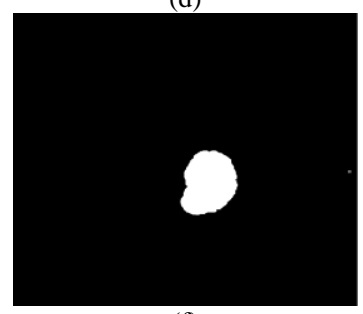

(f)

Figure 4. nucleus segmentation stepwise results of proposed algorithm. (a) Original image (b) 'a' component (c) OTSU thresholded (d) GLCM output (e) morphological filling (f) opening

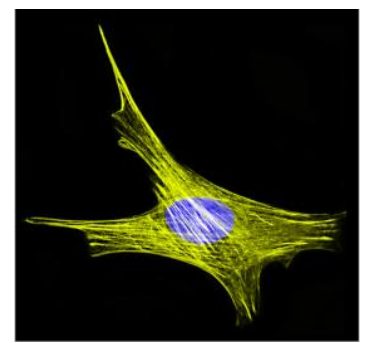

(a)

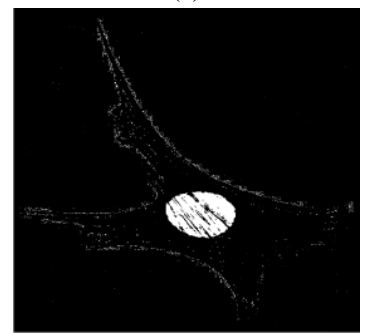

(c)

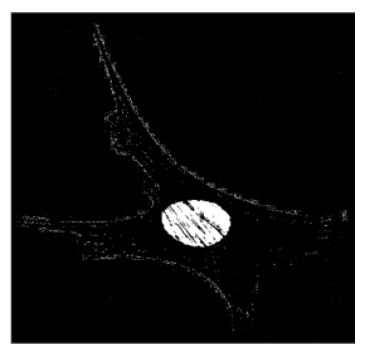

(b)

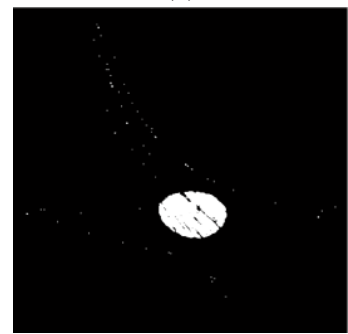

(d) 


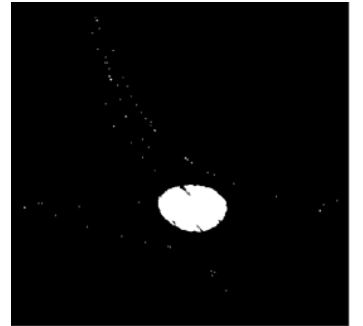

(e)

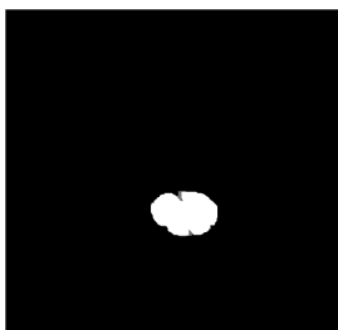

(f)
Figure 5. nucleus segmentation stepwise results of proposed

algorithm. (a) Original image (b) 'a' component (c) OTSU thresholded (d) GLCM output (e) morphological filling (f) opening

\section{CONCLUSION}

The information, which the commonly used grayscale images contain, is not enough for flower segmentation. When flower images are collected in nature, the background is generally more complex and more close to the target color, so there are some limitations in these conditions if we only use gray level information. However, the color images are able to provide more information. There are colors and color depth information, in addition to its provision of brightness and color images can be expressed by a variety of color space. Therefore, segmentation based on color image can overcome some shortcomings of gray-scale image. In this algorithm both color and texture features are considered. In this method, segmentation speed is faster and without human participation, the segmentation result is also ideal. The results show the efficiency of the above algorithm.

\section{ACKNOWLEDGMENT}

The authors would like to express their gratitude to Sri K.V.V. Satya Narayana Raju, Chairman, and K. Sashi Kiran Varma, Managing Director, Chaitanya group of Institutions for providing necessary infrastructure. Authors would like to thank anonymous reviewers for their valuable comments and SRRF members for their invaluable help. The authors would like to express their gratitude to Dr.G.Tulsi Ram Das, Vice chancellor, JNTU Kakinada.

\section{REFERENCES}

[1] S. Belongie, et. al., "Color- and texture-based image segmentation using EM and its application to content-based image retrieval", Proc. of ICCV, p. 675-82, 1998

[2] Y. Delignon, et. al., "Estimation of generalized mixtures and its application in image segmentation", IEEE Trans. on Image Processing, vol. 6, no. 10, p. 1364-76, 1997.

[3] D.K. Panjwani and G. Healey, "Markov random field models for unsupervised segmentation of textured color images", PAMI, vol. 17, no. 10, p. 939-54, 1995.
[4] J.-P. Wang, "Stochastic relaxation on partitions with connected components and its application to image segmentation", PAMI, vol. 20, no.6, p. 61936, 1998.

[5] S.C. Zhu and A. Yuille, "Region competition: unifying snakes, region growing, and Bayes/MDL for multiband image segmentation", PAMI, vol. 18, no. 9, p. 884-900.

[6] L. Shafarenko, M. Petrou, and J. Kittler, "Automatic watershed segmentation of randomly textured color images", IEEE Trans. on Image Processing, vol. 6, no. 11, p. 1530-44, 1997.

[7] W.Y. Ma and B.S. Manjunath, "Edge flow: a framework of boundary detection and image segmentation", Proc. of CVPR, pp 744-49, 1997.

[8] J. Shi and J. Malik, "Normalized cuts and image segmentation", Proc. of CVPR, p. 731-37, 1997.

[9] M. Borsotti, P. Campadelli, and R. Schettini, "Quantitative evaluation of color image segmentation results", Pattern Recognition letters, vol. 19 , no. 8, p. 741-48, 1998 .

[10] D. Comaniciu and P. Meer, "Robust analysis of feature spaces: color image segmentation", Proc. of IEEE Conf. on Computer Vision and Pattern Recognition, pp 750-755, 1997.

[11] Das, M., Manmatha, R., and Riseman, E. M. 1999. Indexing flower patent images using domain knowledge. IEEE Intelligent systems, Vol. 14, No. 5, pp. 24-33.

[12] Nilsback, M. E. and Zisserman, A. 2004. Delving into the whorl of flower segmentation. In the Proceedings of British Machine Vision Conference, Vol. 1, pp. 27-30.

[13] Das, M., Manmatha, R., and Riseman, E. M. 1999. Indexing flower patent images using domain knowledge. IEEE Intelligent systems, Vol. 14, No. 5, pp. 24-33.

[14] Texture Features and KNN in Classification of Flower images. D S Guru, Y. H. Sharath, S. Manjunath RTIPPR,2010.

[15] Anisotropic Diffusion and Segmentation of Colored Flowers. Shoma Chatterjee, IEEE Sixth Indian Conference on Computer Vision, Graphics \& Image Processing, 2008.

[16] J. Weszka, C. Dyer, A. Rosenfeld, “A Comparative Study Of Texture Measures For Terrain Classification" IEEE Trans. SMC-6 (4), pp. 269285, April 1976.

[17] R.W. Conners, C.A. Harlow, “A Theoretical Comparison Of Texture Algorithms", IEEE Trans. PAMI-2, pp. 205-222, 1980.

[18] D.A. Clausi, M.E. Jernigan, "A fast method to determine co-occurrence texture features", IEEE 
Trans on Geoscience \& Rem. Sens., vol. 36(1), pp. 298-300, 1998.

[19] D.A. Clausi, Yongping Zhao, "An advanced computational method to determine co-occurrence probability texture features", IEEE Int. Geoscience and Rem. Sens. Sym, vol. 4, pp. 2453-2455 2002.

[20] A.E. Svolos, A. Todd-Pokropek, "Time and space results of dynamic texture feature extraction in MR and CT image analysis", IEEE Trans. on Information Tech. in Biomedicine, vol. 2(2), pp. 48-54, 1998.

[21] F. Argenti, L. Alparone, G. Benelli, "Fast algorithms for texture analysis using co-occurrence matrices", IEE Proc on Radar and Signal Processing, vol. 137(6), pp.443-448, 1990.

[22] S. Kiranyaz, M. Gabbouj, "Hierarchical Cellular Tree: An Efficient Indexing Scheme for ContentBased Retrieval on Multimedia Databases", IEEE Transactions on Multimedia, vol. 9(1), pp. 102-119, 2007.

[23] A. Baraldi, F. Parmiggiani, “An Investigation Of The Textural Characteristics Associated With GLCM Matrix Statistical Parameters", IEEE Trans. on Geos. and Rem.Sens., vol. 33(2), pp. 293-304, 1995.

[24] R. Haralick, K. Shanmugam, I. Dinstein, "Texture Features For Image Classification", IEEE Transaction, SMC-3(6). Pp. 610-621, 1973.

[25] N. Otsu, "A Threshold Selection Method from Gray- Level Histogram", IEEE Trans. on System Man Cybernetics, vol. 9(I), pp. 62-66, 1979.

[26] P. Gong, J. D. Marceau, and P. J. Howarth, "A comparison of spatial feature extraction algorithms for land-use classification with SPOT HRV data", Proc.Remote Sensing Environ, vol. 40, pp. 137-151, 1992.

[27] A. Ukovich, G. Impoco, G. Ramponi, "A tool based on the GLCM to measure the performance of dynamic range reduction algorithms", IEEE Int. Workshop on Imaging Sys. \& Techniques, pp. 3641, 2005.

[28] http://www.flickr.com

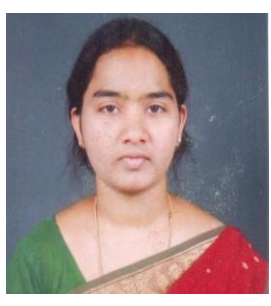

Saka Kezia received the B.Tech (ECE) degree from JNTU College of Engineering, JNT University, Kakinada in 2002. She received M.Tech IIT Madras, India in 2004. She is having nearly 8 years of teaching and industrial experience. She is currently working as Associate Professor, Dept of E.C.E, CIET, Rajahmundry. She is pursuing Ph.D from JNT University, Kakinada in ECE under the guidance of Dr. V. Vijaya Kumar. She is a life member of ISTE, IETE, Red Cross Society and she is a member of SRRF-GIET, Rajahmundry. She has presented 8 papers in various Inter National conferences proceedings. She has published 6 International journal papers.

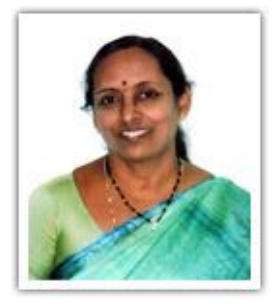

Dr. I. Santhi Prabha is a professor in ECE department and also director of Empowerment of Women \& Grievances at JNTUK, Kakinada. She did her B.Tech in Electronics \& communication engineering from JNTU college of Engineering, Kakinada and Masters Degree in the area of Instrumentation \& controls and Doctoral degree in the field of Speech signal processing from the same institution. She has more than 25 years of experience in teaching. She has published more than 20 papers in various journals and conferences. She is a member of ISTE, IETE and fellow member in Institution of Engineers.

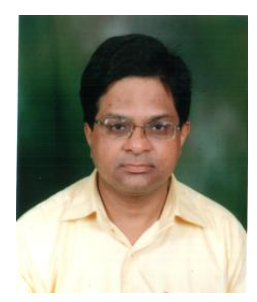

Vakulabharanam Vijaya Kumar received integrated M.S. Engg. , degree from Tashkent Polytechnic Institute Associate Professor and taught courses for M.Tech students. $\mathrm{He}$ has been working as Dean Computer sciences and Head Srinivasa Ramanujan Research Forum-GIET, Rajahmundry, Affiliated to JNT University, Kakinada. His research interests include Image Processing, Pattern Recognition, Network Security, Steganography, Digital Watermarking, and Image retrieval. He is a life member for CSI, ISC, ISTE, IE (I), IRS, ACS, CS and Red Cross. He has published more than 100 research publications in various National, Inter National conferences, proceedings and Journals. 